\title{
Nouvelles datations radiocarbone du Magdalénien de la Chaire-à-Calvin (Mouthiers-sur-Boëme, charente)
}

New Radiocarbon Dates of the Magdalenian from La Chaire-à-Calvin (Mouthierssur-Boëme, Charente)

Neue Radiokarbondatierungen des Magdaleniens von La Chaire-à-Calvin (Mouthiers-sur-Boëme, Charente)

Christophe Delage, Thibaut Devièse et Arnaud Lenoble

\section{OpenEdition \\ Journals}

Édition électronique

URL : http://journals.openedition.org/rao/3614

DOI : 10.4000/rao.3614

ISSN : 1775-3732

Éditeur

Presses universitaires de Rennes

Édition imprimée

Date de publication : 31 décembre 2017

Pagination : 11-22

ISBN : 978-2-7535-7540-0

ISSN : 0767-709X

\section{Référence électronique}

Christophe Delage, Thibaut Devièse et Arnaud Lenoble, « Nouvelles datations radiocarbone du Magdalénien de la Chaire-à-Calvin (Mouthiers-sur-Boëme, charente) », Revue archéologique de l'Ouest [En ligne], 34 | 2017, mis en ligne le 13 juin 2020, consulté le 25 février 2021. URL : http:// journals.openedition.org/rao/3614; DOI : https://doi.org/10.4000/rao.3614 


\title{
Nouvelles datations radiocarbone du Magdalénien de la Chaire-à-Calvin (Mouthiers-sur-Boëme, Charente)
}

\author{
New Radiocarbon Dates of the Magdalenian from La Chaire-à-Calvin \\ (Mouthiers-sur-Boëme, Charente)
}

\author{
Christophe Delage ${ }^{a}$ Thibaut Devièse ${ }^{b}$ et Arnaud Lenoble ${ }^{c}$
}

\begin{abstract}
Résumé : Le gisement magdalénien de La Chaire-à-Calvin (Charente) est connu de longue date. Toutefois, après 150 ans de recherche, les interprétations chrono-stratigraphiques, avancées par plusieurs générations de chercheurs, ne s'accordent pas entre elles, ni avec les deux datations radiocarbone disponibles. Dans ce contexte, un nouveau programme de mesures radiométriques du remplissage a abouti à trois nouvelles dates. La présente note expose ces résultats et en discute la validité au sein de la séquence du site.
\end{abstract}

Abstract: The Magdalenian site of La Chaire-à-Calvin (Charente) has been known of for a long time. However, after 150 years of research, the many chrono-stratigraphic interpretations put forward by generations of scholars do not concur with each other nor with the two available radiocarbon dates. A new program of radiometric measurements of the filling has provided three new dates. The present note details these results and discusses their value within the archaeological sequence of the site.

Mots clés : La Chaire-à-Calvin, Magdalénien, datations radiocarbone

Keywords: La Chaire-à-Calvin, Magdalenian, radiocarbon dating

\section{INTRODUCTION}

Le gisement archéologique de La Chaire-à-Calvin est situé le long du Gersac, petit ruisseau qui se jette dans la Boëme, affluent de la Charente, sur la commune de Mouthiers-surBoëme (Charente). Il s'agit d'un petit abri-sous-roche qui s'ouvre vers le sud. Il semble que ce soit le principal fouilleur des lieux, P. David, qui lui ait trouvé ce nom, en inventant de toute pièce une légende qui relaterait la présence de J. Calvin sur place pour prêcher sa réforme religieuse (Delage et Porte, 2015).
Cet abri aurait été découvert peut-être par P. de Vibraye en 1863, puis exploré succinctement par A. Trémeau de Rochebrune l'année suivante (Trémeau de Rochebrune, 1865). C'est ensuite entre 1924 (ou en 1926) et 1933 que les premières fouilles d'ampleur furent menées par P. David. Le moment fort de cette phase de terrain fut la découverte de la frise sculptée sur la paroi de l'abri (David, 1928; 1929; 1963). Après la Seconde Guerre mondiale, P. David y reprit ses recherches de 1947 à 1959 (David, 1957). La quasitotalité du site a ainsi été fouillée : la plus grande partie de l'abri et le talus qui le prolonge. À l'invitation de P. David,

a'Département de Préhistoire, Muséum National d'Histoire Naturelle, Paris. (delage.chris@laposte.net)

${ }^{\mathrm{b}}$ Research Laboratory for Archaeology and the History of Art, Oxford University, Oxford, United Kingdom. (thibaut.deviese@arch.ox.ac.uk)

'PACEA-CNRS UMR 5199 - Université de Bordeaux, Allée Geoffroy-Saint-Hilaire, Pessac. (arnaud.lenoble@u-bordeaux.fr) 
D. de Sonneville-Bordes et F. Bordes y entreprirent de brèves explorations en 1960 et 1961 (de Sonneville-Bordes, 1987). Trois mètres carrés du témoin préservé à l'extrémité orientale du site furent alors prélevés. Les dernières fouilles d'envergure, dirigées par J.-M. Bouvier, eurent lieu de 1966 à 1972 (Bouvier, 1969; 1976; Bouvier et Debénath, 1969) et consistèrent à réaliser une fouille repoussant sur deux mètres le témoin est, de l'abri jusqu'au pied de talus. Ce gisement fit également l'objet dernièrement d'études ponctuelles sur le terrain. Tout d'abord, entre 2005 et 2008, G. Pinçon et son équipe effectuèrent de nouveaux relevés (notamment en 3D) et observations de l'ensemble sculpté (cf. Pinçon et al., 2008; Bourdier et al., 2008; Pinçon et Bourdier, 2009). Ensuite, à la fin de l'été 2009, un nettoyage de la coupe stratigraphique sagittale (fouilles Bordes et Bouvier) permit d'effectuer - entre autres - de nouvelles observations géoarchéologiques (Delage, 2010; Delage et al., 2010).

Dans la mesure où ce gisement a été découvert et exploré très tôt, au cours de la première phase des recherches préhistoriques ( $\mathrm{du}$ milieu $\mathrm{du} \mathrm{XIX}^{\mathrm{e}}$ siècle au début du $\mathrm{xx}^{\mathrm{e}}$ siècle), il a souffert de techniques de fouilles anciennes et de méthodes de collecte du matériel peu rigoureuses. Par contre, les fouilles de F. et D. Bordes et de J.-M. Bouvier, réalisées selon des méthodes modernes, ont livré des vestiges archéologiques dans des contextes stratigraphiques mieux précisés. Ces derniers chercheurs insistent sur le fait que le remplissage et les habitats préhistoriques sont homogènes et cohérents, et qu'ils ne peuvent " correspondre qu'au Magdalénien supérieur et même final " (Bouvier, 1969, p. 2865 ; cf. de Sonneville-Bordes, 1987 ; Debénath, 2006, p. 315). Pour se justifier, ils critiquent les éléments traditionnellement avancés à cette époque pour caractériser le Magdalénien inférieur-moyen (et qui caractérisent en fait les niveaux de leurs propres fouilles à la Chaireà-Calvin) : présence de triangles scalènes, de Sä̈ga tatarica, absence de harpons; et ils ne tiennent pas compte de la seule datation radiocarbone disponible à l'époque, qui va également à l'encontre de leurs interprétations (voir ci-dessous). Ces interprétations perdurent encore dans la littérature (cf. Debénath, 2006; 2014), malgré les efforts menés notamment par F. Delpech, P.-Y. Demars et F. Djindjian, pour les réfuter et y substituer une attribution au Magdalénien moyen ancien.

Malgré la qualité de ces derniers travaux, et à l'encontre des interprétations de ces auteurs, la chronologie des occupations humaines paléolithiques de ce site - notamment celles du Magdalénien qui nous intéressent ici - est entourée de la plus grande confusion et, dès lors, est loin d'être résolue de manière satisfaisante. Dans cet article, nous mettons l'accent sur la question de la datation et de la chronologie absolue de la séquence magdalénienne de la Chaire-à-Calvin. Dans un premier temps, nous présentons les différentes interprétations stratigraphiques qui se sont succédées au cours de la longue période de recherche qu'a connue le site, tout en nous efforçant de cerner leurs limites et les incertitudes à prendre en compte dans l'interprétation des résultats chronologiques. Ensuite, nous exposons et discutons les premières datations radiométriques obtenues. Enfin, nous livrons les résultats d'un récent programme de datations radiocarbone.

\section{Stratigraphie DU SITE}

Le nettoyage de coupe pratiqué en 2009 concerne la coupe sagittale laissée par J.-M. Bouvier à l'issue de ses fouilles (Delage et al., 2010). Cette section, longue d'environ $6 \mathrm{~m}$, documente un dépôt d'une puissance d'1,5 m préservé sur la terrasse rocheuse s'étendant sur trois mètres au-devant de l'abri, ainsi que le talus où le dépôt atteint une puissance de 2,5 m (fig. 1A). Dans l'abri, cette coupe est prolongée par le témoin de P. David sur les sections duquel se lisent les grandes involutions de cailloutis et les poches de limons dans lesquelles $\mathrm{H}$. Alimen a reconnu un sol polygonal trié (Alimen et David 1949; Alimen 1950).

Une première lithostratigraphie a été proposée par P. David (1957). Le remplissage y est subdivisé en 5 unités lithostratigraphiques d'épaisseur hémimétrique. Les dépôts présents au-devant de l'abri ont fait l'objet d'une proposition de découpage stratigraphique plus détaillé par D. de Sonneville-Bordes (1965), sur la base des variations de taille et d'abondance de la fraction de granules et de cailloux calcaires. Six couches y sont distinguées en lieu et place de la seule couche 2 de P. David. Les mêmes critères sédimentaires ont été utilisés par J.-M. Bouvier et A. Debénath (1969) et les 6 niveaux archéologiques précédemment définis ont été maintenus et, parfois, subdivisés (couches 5 et 6). L'extension de ces niveaux reste toutefois limitée aux premiers mètres de la terrasse, du fait des fortes variations latérales de faciès au niveau du talus.

La lecture stratigraphique réalisée en 2009 s'est basée sur la notion d'unité lithostratigraphique définie comme " un corps tridimensionnel caractérisé par la présence généralisée d'une dominante d'un certain type de lithologie ou par la combinaison de deux ou plusieurs de ces types [...] conférant à ladite unité un caractère d'homogénéité " (Gasche et Tunca, 1981). Les variations lithologiques de détail ayant prévalu à la définition des couches des précédents auteurs n'ont pas été retenues comme pertinentes, dans la mesure où elles peuvent ne représenter que l'expression de processus agissant plus ou moins simultanément ou en relais, lorqu'elles ne sont pas seulement issues de ségrégations gra- 

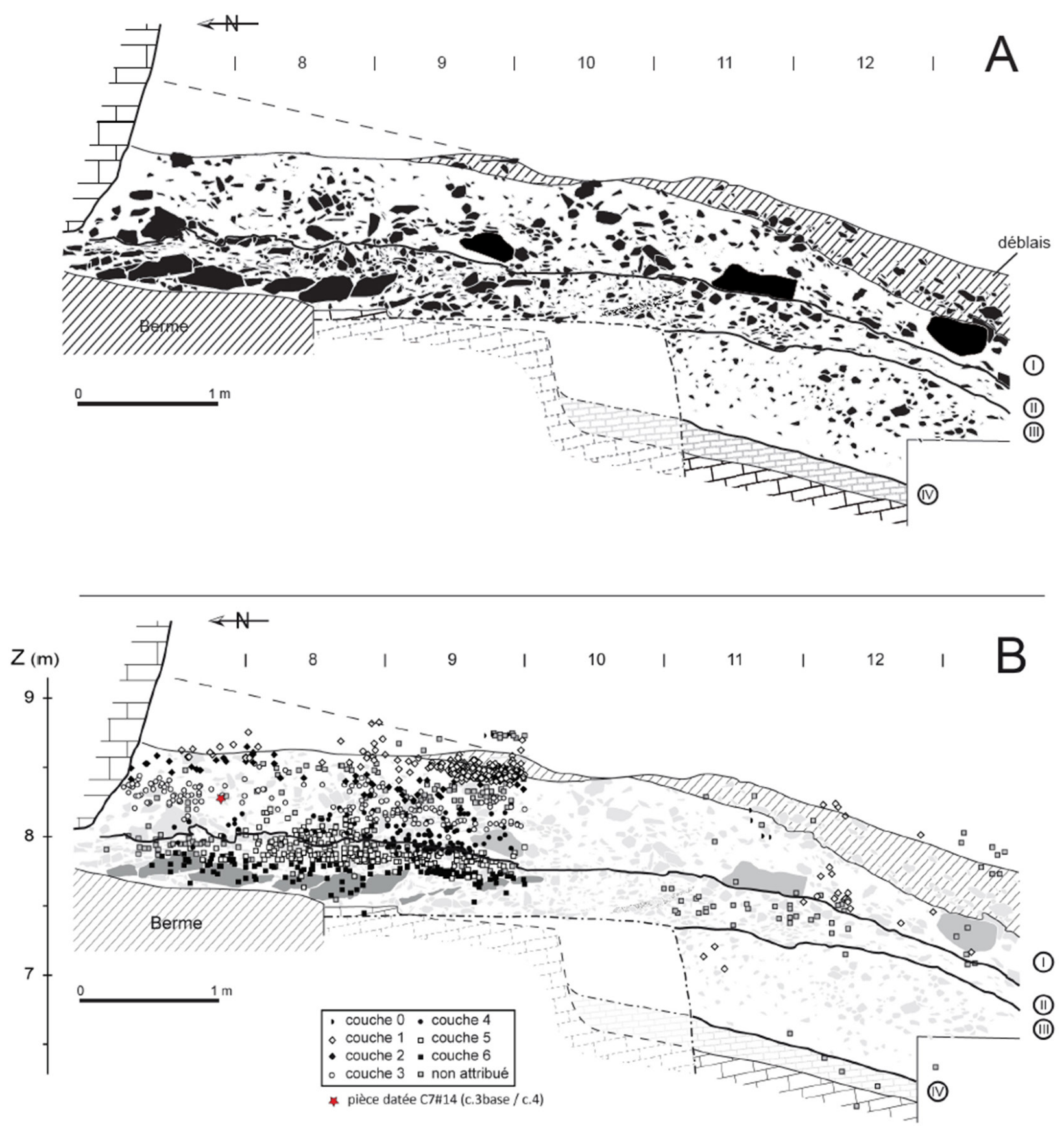

Figure 1 : Relevé de la coupe nettoyée du témoin est (A). Les unités lithostratigraphiques sont indiquées par les chiffres romains cerclés. (B) Report sur fond de levée de coupe des vestiges côtés sur le mètre de la fouille Bouvier précédant le témoin. La pièce datée de la couche 4 y est localisée (étoile rouge).

Figure 1: Drawing of the section in the East Slope Area (A). Roman numbers designate our stratigraphic units. (B) Distribution of artifacts surimposed over the stratigraphic drawing. The dated equid tooth from layer 4 is positioned (red star).

nulométriques liées à un même épisode de dépôt (cf. Bertran et Texier, 1999; Texier, 2000).

Sur cette base, quatre unités stratigraphiques ont été reconnues sous le dépôt de déblais modernes (fig. 1A). Trente à quarante centimètres de plaquettes disposées conformément entre elles couvrent le rocher au niveau du talus (Unité IV), issues de la fracturation in situ du calcaire turonien. Soixante-quinze centimètres d'un dépôt massif à débris calcaires émoussés dispersés dans une matrice d'argile limoneuse jaune-rougeâtre riche en granules calcaires (Unité III) s’y superposent. Ce dépôt, adossé à la marche du rocher délimitant la terrasse de l'abri, indique une accu- 
mulation en relation avec une première phase de creusement de l'abri. Il est lui-même recouvert par un demi-mètre d'un cailloutis hétérométrique à débris calcaires sub-anguleux dont la principale caractéristique est une orientation isotrope des cailloux parfois regroupés en cellules de cryoturbation (Unité II). Le colmatage interstitiel est une argile brune massive dont l'observation en lames minces révèle un entassement compact d'ovoïdes cryogéniques à coiffes asymétriques rattachable à un horizon supérieur de cryosol (Van Vliet-Lanoë, 2005). Cette unité prolonge le témoin préservé dans l'abri et l'on pense que l'absence de sédiment fin, ne permettant plus une expression aussi nette du sol polygonal trié décrit par Alimen, est liée à une éluviation des dépôts situés à l'extérieur de l'abri. Cette unité contient les niveaux archéologiques 5 et 6 de Bouvier. Les caractéristiques granulométriques des cailloux ayant conduit à l'individualisation de ces deux couches et de leurs subdivisions (e.g. couche 6-2, poches sablo-argileuses comblant des cellules de convention; Bouvier et Debénath, 1969) ne représentent vraisemblablement qu'une ségrégation des éléments par cryoexpulsion et cryoreptation lors de la formation du cryosol (cf. Van Vliet-Lanoë 2005). Cette unité est recouverte d'un dépôt d'égale puissance dont le faciès général est celui d'un diamicton à litage lenticulaire mal exprimé au-devant de l'abri et d'un dépôt non organisé sur le talus (Unité I). Ces deux faciès sont disposés en relais et aucune discordance ne permet d'inférer un emboîtement de ces deux termes, contra le schéma stratigraphique publié par Bouvier (Bouvier et Debénath, 1969). Cette unité contient des cailloux calcaires anguleux de toutes tailles, localement regroupés en lentilles subhorizontales aplaties à support clastique d'extension métrique à inframétrique, qui ont été reconnues comme autant de niveaux archéologiques par
D. de Sonneville-Bordes, et Bouvier à sa suite (couches 1 à 4). Les débris sont disposés plutôt conformément au plan de stratification; aucun trait cryogénique n'est observé en lames minces, et cette unité s'individualise par la présence, aussi bien dans la fraction figurée que dans la fraction fine, d'éléments siliceux (e.g. fragments de dalles silico-ferrugineuses, quartzite sédimentaire) dérivés des altérites couvrant les plateaux du secteur (Bourgueil et al., 1970). Quelques figures de tris observées en lames minces et les lentilles de granules calcaires localement observées (e.g. couche B de Bouvier et Debénath, 1969) témoignent d'une contribution du ruissellement à l'édification des dépôts (Lenoble, 2005), tandis que l'ébauche d'un litage par ségrégation de lentilles caillouteuses, la morphologie et l'extension de ces lentilles, suggèrent que des épisodes de coulée de débris ont également contribué à la formation de cette unité (Bertran et Coussot, 2004). Ces dynamiques sédimentaires, conjuguées à la remobilisation des formations de plateau et la morphologie en talus à double pente de cette unité en section sagittale, permettent d'y reconnaître un cône colluvial nourri par les dépôts de pente.

Le tableau 1 donne les équivalences entre cette relecture stratigraphique et les découpages réalisés auparavant. Il en ressort deux principaux ensembles archéologiques. L'un, supérieur, correspond à l'unité I à laquelle se rattachent grosso modo les niveaux archéologiques 1 à 4 de Bouvier. Lautre, inférieur, correspond à l'unité II à laquelle se rattachent grosso modo les couches 5 et 6 de Bouvier. Le caractère grossier des rattachements tient aux difficultés que soulève une confrontation de détail entre les différentes stratigraphies.

Ainsi, les limites entre les unités lithostratigraphiques que nous proposons peuvent être suivies sur l'ensemble des

\begin{tabular}{|c|c|c|c|c|c|}
\hline \multirow{2}{*}{ David, 1947} & \multirow{2}{*}{$\begin{array}{c}\text { Sonneville- } \\
\text { Bordes, } 1963\end{array}$} & \multicolumn{3}{|c|}{ Bouvier et Debenath, 1969} & \multirow{2}{*}{$\begin{array}{c}\text { lithostratigraphie } \\
2009 \\
\end{array}$} \\
\hline & & lithostratigraphie & \multicolumn{2}{|c|}{ archéostratigraphie } & \\
\hline 1 & 1 & A & \multicolumn{2}{|c|}{$\begin{array}{c}0 \\
\text { "dallage intentionnel" }\end{array}$} & déblais \\
\hline \multirow{4}{*}{2} & 1 & \multirow{2}{*}{ B } & \multicolumn{2}{|c|}{1} & \multirow{5}{*}{ U1 } \\
\hline & 2 & & & & \\
\hline & 3 & \multirow{2}{*}{ C } & \multirow{2}{*}{\multicolumn{2}{|c|}{3}} & \\
\hline & 4 & & & & \\
\hline 3 & 5 & $/ \mathrm{E}$ & 4 & 5 & \\
\hline \multicolumn{6}{|c|}{ CRYOSOL } \\
\hline \multirow{4}{*}{4} & \multirow{2}{*}{6} & \multirow{2}{*}{$E$} & & $5-1$ & \multirow{4}{*}{ U2 } \\
\hline & & & & $5-2$ & \\
\hline & \multirow{5}{*}{ non vu } & $\mathrm{F}$ & \multirow{2}{*}{6} & $6-1$ & \\
\hline & & G & & $6-2$ & \\
\hline 5 & & $\mathrm{H}$ & \multicolumn{2}{|c|}{ stérile } & U3 \\
\hline 6 & & \multirow{2}{*}{ I } & \multirow{2}{*}{\multicolumn{2}{|c|}{ Rocher }} & $\mathrm{U} 4$ \\
\hline Rocher & & & & & Rocher \\
\hline
\end{tabular}

Tableau 1 : Équivalence entre les stratigraphies des différentes fouilles. Le sommet du cryosol est situé, selon notre interprétation, pour information, bien qu'il n'ait pas été reconnu comme tel par tous les auteurs. Table 1: Correlative table of stratigraphies of the different authors. The summital surface of the cryosol is shown for information, following our own interpretation, even though this feature was not recognized by all authors. 
témoins et ont valeur d'isochrones. Pourtant, le report des projections des pièces côtées lors des fouilles Bouvier sur fond de levée stratigraphique montre que ces contacts n'ont pas toujours été suivis à la fouille (fig. 1B). Cela tient probablement à la définition granulométrique des couches lors des anciennes fouilles pour lesquelles du sédiment d'unités différentes, mais à texture proche, a été associé dans une même couche. Le résultat en est que certaines couches associent des pièces de différentes unités, à l'exemple des couches 4 et 5 regroupant chacune des pièces provenant des unités I et II et, qu'à l'inverse, des pièces issues d'une même nappe de vestiges sont partagées entre différentes couches (fig. 1B). Un autre soucis tient à la définition strictement granulométrique des couches contenues dans l'unité II, alors que les lentilles correspondantes apparaissent d'extension très limitée. Le risque en est que deux lentilles distinctes, mais de texture comparable, soient regroupées dans un même ensemble archéologique et, qu'à l'inverse, des éléments d'une même nappe de vestiges contenus dans des sédiments de faciès distincts aient été affectés à différents " ensembles archéologiques ». Cette dernière hypothèse trouve une belle illustration dans la mise en vis-à-vis des pièces côtées lors des fouilles de D. de Sonneville-Bordes et de J.-M. Bouvier (fig. 2). Quand bien même les couches des deux auteurs ont la même définition lithologique, un décalage apparaît où la couche $2 \mathrm{de} \mathrm{J}$.-M. Bouvier vaut la couche 1 de $\mathrm{D}$. de Sonneville-Bordes, la couche 3 de J.-M. Bouvier la couche 2 de D. de Sonneville-Bordes, et ainsi de suite jusqu'à la base du remplissage.
Ce dernier point implique de bien identifier les fouilles dont sont issues les pièces datées. Le premier problème, en revanche, à savoir la partition de nappe de vestige entre différentes couches et l'association dans une même " couche » de vestiges issus de différentes nappes, voire de différentes unités lithostratigrahpiques, est source de nombreuses incertitudes que nous nous sommes efforcés de prendre en compte dans l'interprétation des résultats radiochronologiques.

\section{Commentaires Sur les datations ABSOLUES PRÉCÉDENTES}

Deux datations radiocarbone ont été effectuées précédemment sur du matériel archéologique de La Chaire-à-Calvin. Un premier échantillon constitué de plusieurs fragments d'os, collecté en 1969 par J.-M. Bouvier dans le niveau archéologique 6, fut soumis à Jacques Evin (Laboratoire de datation par le radiocarbone de Lyon) en 1978, afin de réaliser une première datation radiométrique. Le matériel fourni permit d'obtenir la date suivante (non calibrée) : $15440 \pm$ 440 B.P. (Ly-1998) (Evin et al., 1983, p. 115-116) ; c'est-à-

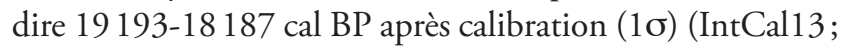
Reimer et al., 2013).

Une nouvelle mesure radiocarbone, obtenue par la méthode de la Spectrométrie de Masse par Accélérateur (SMA), fut réalisée dans le cadre d'un programme de datations de sites paléolithiques et épipaléolithiques anciennement fouillés du Poitou-Charentes (2000-2002) dirigé
Figure 2 : Projection de la bande 8 du témoin Est mettant en vis-àvis les fouilles Sonneville-Bordes et Bouvier. Ce graphique illustre la non-congruence de numérotation des couches archéologiques entre ces deux fouilles.

Figure 2: Vertical distribution of artifacts along the line 8 of the squaregrid aligning Sonneville-Bordes' and Bouvier's excavated squares. This plot illustrates the discrepancy between the layers of each excavator.

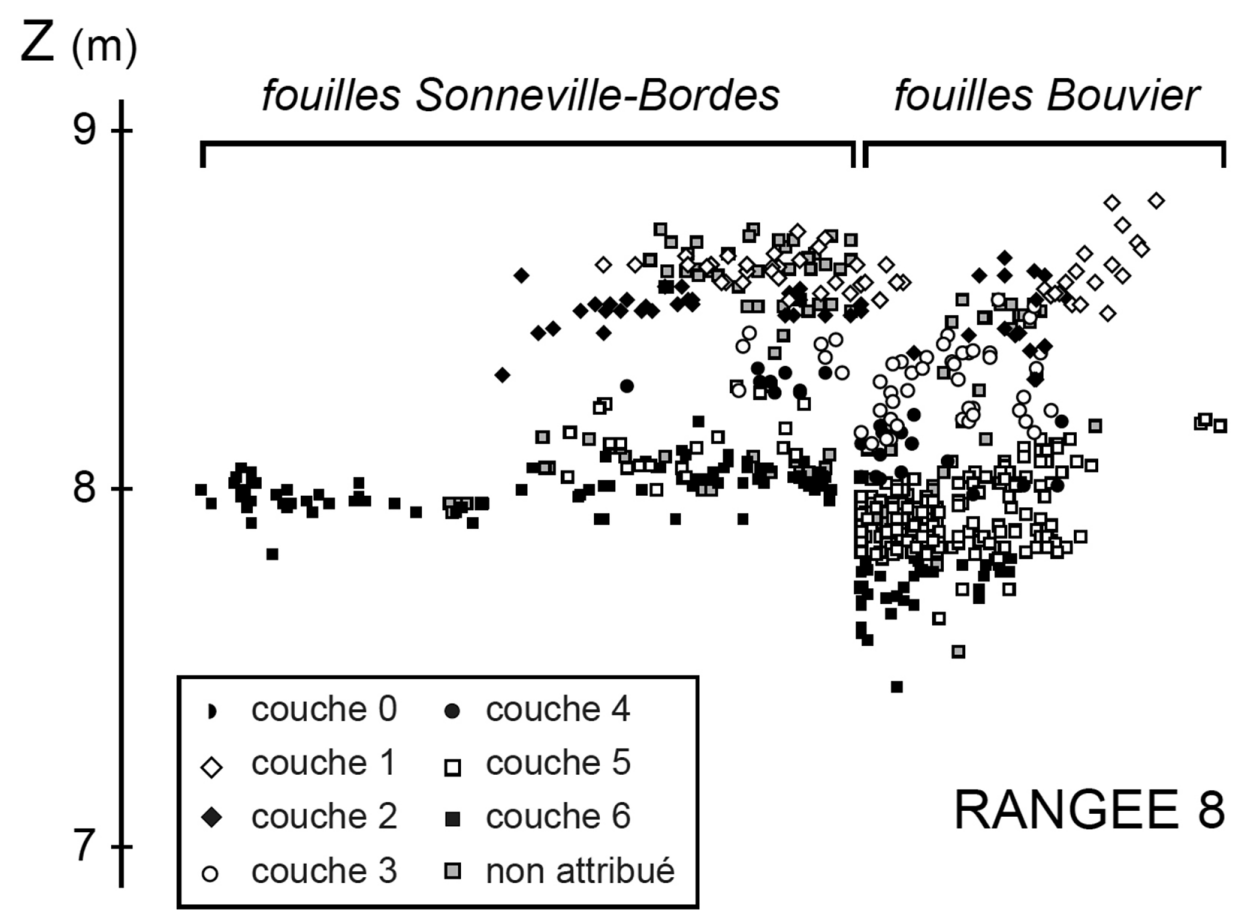


par V. Dujardin. Cette seconde date, effectuée sur un os d'Antilope saïga et provenant également du niveau 6 des fouilles Bouvier, est de $16020 \pm 80$ B.P. (non calibré) (OxA12053/Ly-2098) (Dujardin et Tymula, 2005, fig. 1, p. 785), ce qui donne par calibration 10 (IntCal13) : 19466-19216 cal. BP. Dans le cadre de ce même projet de recherche, V. Dujardin avait soumis un autre échantillon d'os, provenant de la couche 1 des fouilles de D. de Sonneville-Bordes et F. Bordes (1960-1961), mais cet échantillon s'est avéré sans collagène (cf. Dujardin et Tymula, 2005, fig. 1, p. 785).

Que dire de ces deux dates? Elles ont été obtenues, à plus de trente ans d'écart, par deux chercheurs différents, qui avaient des motivations et des objectifs différents. Véronique Dujardin s'est intéressée, entre autres, à mieux cerner chronologiquement la présence de l'Antilope saïga dans la région Poitou-Charentes. À cette fin, elle a sélectionné pour datation radiométrique des échantillons osseux de cette espèce en provenance de sites différents du Paléolithique supérieur. Mais sa problématique n'était pas liée précisément à la chronologie de chacun des gisements sélectionnés.

La situation était différente pour J.-M. Bouvier. En 1978, au moment où il soumit un échantillon osseux pour datation, J.-M. Bouvier avait achevé depuis quelques années déjà ses fouilles à La Chaire-à-Calvin - et il était proche de la fin (1980) de celle de La Madeleine (Bouvier, 1977) - mais, surtout, il avait une idée bien précise de la stratigraphie et de la chronologie de ce gisement, ainsi que de sa place au sein de la "civilisation " magdalénienne du sud-ouest de la France (cf. Bouvier, 1969). En s'appuyant sur les observations sédimentologiques du remplissage effectuées par A. Debénath, ainsi que celles sur la faune (F. Prat) et les silex taillés (D. de Sonneville-Bordes), J.-M. Bouvier évoquait une "sédimentation très rapide ", convaincu de la " très grande homogénéité " des industries et de la " relative briéveté de l'occupation ", attribuable au Magdalénien supérieur (voire final) (Bouvier, 1968, p. 71; 1969 ; 1971, p. $165 ; 1976$, p. $134 ; 1985$, p. 182 ; Bouvier et Debénath, 1969, p. 215; Debénath, 1974; 2006, p. 233). Une telle datation (relative) pouvait alors se justifier parce que " la confusion des niveaux... est moins préjudiciable qu'en un autre gisement " (Bouvier, 1971, p. 165). Dans ce contexte, Bouvier ne pouvait accepter la date obtenue par J. Evin; il considérait qu'elle était bien plus ancienne que prévu (in Evin et al., 1983, p. 115). Cette mesure radiométrique était pourtant comparable à deux autres, inédites, provenant de Saint-Germain-la-Rivière (Gironde). Qui plus est, l'industrie lithique de ce dernier gisement semblait similaire à celle de la Chaire-à-Calvin (couche 6). Ces correspondances et commentaires étaient le fait de Bouvier lui-même, publiés à la suite de la datation (in Evin et al., 1983, p. 115). Ces différents éléments auraient pu paraitre troublants et ques- tionner Bouvier. Au contraire. Ces faisceaux d'indices ne s'accordaient pas avec le modèle d'évolution des sociétés magdaléniennes qu'il avait proposé une dizaine d'années plus tôt, dans lequel un faciès sans harpon se serait développé au cours du Magalénien supérieur dans la région Poitou-Charentes (Bouvier, 1969). Il écarta donc cette datation, et ne la mentionnera à nouveau que beaucoup plus tard (Bouvier et Crémadès, 1989, p. 78-79), sans en tirer d'implications chrono-culturelles. De même, Debénath allait faire corps avec son collègue de longue date et aucune de ses récentes publications (2006, p. 229-237; 2014, p. 250-255) ne mentionnait cette datation ni de quelconque problème de chronologie interne au gisement.

Depuis les années 70, la datation par le carbone 14 s'est beaucoup affinée et les recherches sur le Magdalénien ont beaucoup progressé. Divers chercheurs (e.g. F. Delpech, P.-Y. Demars, F. Djindjian), en s'appuyant sur des approches variées, ont ré-interprété la séquence de ce gisement, attribuant ses niveaux inférieurs (au moins) à la phase moyenne (ou inférieure) du Magdalénien. La datation issue du projet Dujardin conforte cette proposition. Par contre, nous doutons sérieusement de la fiabilité de la date Evin/Bouvier, de $15440 \pm 440$ B.P. Tout d'abord, elle a été effectuée anciennement, selon la méthode conventionnelle de datation radiocarbone, induisant des incertitudes de mesures relativement importantes; l'écart-type est ainsi large : 440 ans. Ensuite, elle a été réalisée à partir de plusieurs ossements; elle représente de ce fait une moyenne à partir de pièces qui pouvaient être d'âge assez différent. Cette datation ancienne est donc à considérer avec prudence.

\section{NOUVEAU PROGRAMME DE DATATIONS RADIOMÉTRIQUES}

Pour sortir de cette impasse, il était impératif de s'appuyer sur de nouvelles datations " absolues». Un programme de mesures radiométriques a donc été mis sur pied en 2009 afin d'établir un cadre chrono-stratigraphique plus solide. Cinq dates C14-SMA du remplissage et des occupations préhistoriques ont alors été obtenues.

\section{Matériel}

Les échantillons sélectionnés pour ce projet proviennent des fouilles Bouvier archivées dans les collections archéologiques du Musée des Beaux-Arts d'Angoulême. Laure Fontana a mis de côté certains échantillons osseux déterminés anatomiquement et taxonomiquement lors de son premier examen de l'assemblage faunique, en juillet 2008. 
Nous avons sélectionné cinq échantillons osseux correspondant aux niveaux 5 à 1 , en suivant la stratigraphie de bas en haut. Le niveau archéologique 6-2 étant très limité, nous ne sommes pas convaincus qu'il soit pertinent de le dater (pour l'instant au moins). Le niveau archéologique 6 a déjà fourni deux datations radiométriques, qui permettent de le situer chronométriquement, Le niveau archéologique 5 (qui inclut les niveaux archéologiques 5-1 et 5-2) fait ici l'objet d'une nouvelle datation, réalisée sur un os de renne. Il en est de même des quatre niveaux archéologiques sus-jacents (4 à 1) (tableau 2).

Il est à remarquer que cette sélection de pièces a été faite avant que la saisie des carnets de fouille et leur confrontation à la relecture stratigraphique du site n'aient été réalisées; raison pour laquelle les difficultés liées au découpage stratigraphique de J.-M. Bouvier n'étaient pas identifiées (cf. $\$ 2$. Stratigraphie). De fait, aucun caractère de priorité n’a alors été donné au fait que les pièces retenues pour datation relèvent des quelques pièces osseuses côtées lors de la fouille (5,4\% des objets côtés sont des restes osseux ou dentaires). Aussi, n'est-on pas en capacité de porter toutes les pièces datées sur les projections et, par là-même, de s'assurer de leur position stratigraphique véritable. Les incertitudes qui en résultent sont détaillées dans la discussion.

\section{Méthodologie}

Les cinq échantillons d'os sélectionnés ont suivi un traitement afin d'extraire le collagène tel qu'il est décrit par Brock et al. (2010). Ces échantillons ont, dans un premier temps, été sablés avec de la poudre d'oxyde d'aluminium afin de nettoyer leur surface; puis ils ont été broyés à l'aide d'un pilon et un mortier en acier. Ils ont ensuite été déminéralisés par trois traitements avec de l'acide chlorhydrique $(0,5 \mathrm{M})$. Après la déminéralisation, la fraction organique a été rincée trois fois avec de l'eau déminéralisée ultrapure MilliQ ${ }^{\mathrm{TM}}$. Les échantillons ont ensuite été traités avec de l'hydroxyde de sodium $(0,1 \mathrm{M})$ pour éliminer d'éventuels contaminants humiques, puis ont été de nouveau rincés trois fois avec de l'eau déminéralisée ultrapure MilliQ ${ }^{\mathrm{TM}}$. Un lavage final avec de l'acide chlorhydrique $(0,5 \mathrm{M})$ a été effectué pour éliminer tout dioxyde de carbone atmosphérique dissous lors du traitement avec l'hydroxyde de sodium. Encore une fois, ceci a été suivi par trois rinçages avec de l'eau déminéralisée ultrapure MilliQ ${ }^{\mathrm{TM}}$. Pour chaque échantillon, le collagène résultant a été gélatinisé à $75^{\circ} \mathrm{C}$ pendant 20 heures dans une solution d'acide chlorhydrique ( $\mathrm{pH} 3)$, filtré à l'aide d'un Ezee-filter ${ }^{\mathrm{TM}}$, puis transféré dans un ultra-filter (Sartorius Vivaspin $^{\text {TM }} 1530 \mathrm{kDa}$ MWCO). Après centrifugation, la fraction dans le filtre a été lyophilisée pendant 24 heures en utilisant un lyophilisateur Vaco-5 (Zirbus, Allemagne).
La mesure des isotopes stables du carbone et de l'azote, la combustion et la graphitisation ont été effectuées pour chaque échantillon comme décrit dans l'article publié par Brock et al. (2010). Enfin, les trois échantillons ayant produit suffisamment de collagène ont été mesurés par SMA au laboratoire de datation radiocarbone d'Oxford (Oxford Radiocarbon Accelerator Unit) pour quantifier les différents isotopes du carbone.

\section{Résultats}

Sur les cinq échantillons osseux soumis, trois mesures ont pu être effectuées avec succès, les deux autres échantillons concernant les couches 2 et 3 s'avérant sans collagène.

Le tableau 2 présente les résultats de ce nouveau programme de datations radiométriques. Les dates indiquées sont les dates conventionnelles exprimées en années d'après Stuiver et Polach (1977) et les dates calibrées en utilisant IntCal13. Les erreurs standard sont calculées à $1 \sigma$ et $2 \sigma$ (Ramsey et al., 2004). Les rapports des isotopes stables sont exprimés en \%o en rapport à VPDB avec une précision spectrométrique de $\pm 0,2 \%$ o pour $\mathrm{C}$ et $\pm 0,3 \%$ pour $\mathrm{N}$ (Coplen, 1994). C:N est le rapport atomique du carbone sur l'azote et il est acceptable entre 2,9 et 3,5. La cote de la pièce, lorsqu'elle existe, est également portée dans ce tableau.

\section{DisCUSSION}

\section{Niveau 5}

La nouvelle datation, de $15805 \pm 80$ BP (19158$18945 \mathrm{cal}$. BP à $1 \sigma$ ), obtenue pour la couche 5 , nous semble tout à fait cohérente, et ce, pour plusieurs raisons. Elle est très proche et s'accorde bien avec une autre produite récemment pour le niveau sous-jacent : $16020 \pm 80$ BP (Dujardin et Tymula 2005). Ce faible écart montre, en cela, que la base de la séquence livre des industries d'âge comparable. Notre relecture de la lithostratigraphie, un premier essai de corrélation entre les différentes séquences archéostratigraphiques des précédents fouilleurs, et la projection verticale des objets inventoriés et côtés dans les carnets de fouilles, nous révèlent que ces niveaux 6 et 5 relèvent d'un même ensemble lithostratigraphique, à la base de la stratigraphie, pris dans un niveau à fines plaquettes et altéré ultérieurement par la cryoturbation (cf. $\$ 2$. Stratigraphie). Le faible écart entre les deux âges radiocarbone de la base du remplissage et la réinterprétation stratigraphique du site convergent ainsi pour indiquer que la base de la séquence prélevée dans le témoin Est du site se situe autour de 16000 B.P. (ou 19600-19000 cal. BP). 


\begin{tabular}{|c|c|c|c|c|c|c|c|c|}
\hline $\begin{array}{c}\mathbf{N}^{\circ} \\
\text { échantillon }\end{array}$ & \begin{tabular}{|c|} 
Contexte \\
archéologique
\end{tabular} & Matériel & Soumission & Date B.P. & $\begin{array}{c}\text { Date calibrée (B.C.) } \\
\text { (IntCal13) }\end{array}$ & $\delta^{13} \mathbf{C}$ & $\delta^{15} \mathbf{N}$ & C:N \\
\hline $\begin{array}{l}\text { OxA- } \\
22327\end{array}$ & $\begin{array}{c}1 \text { (fouilles } \\
\text { Bouvier) }\end{array}$ & $\begin{array}{l}\text { dent } \\
\text { d'équidé }\end{array}$ & $\begin{array}{l}\text { collecté par J.-M. } \\
\text { Bouvier (fouilles 1966- } \\
\text { 1972) ; sélectionné et } \\
\text { soumis par L. Fontana en } \\
\text { octobre 2009 }\end{array}$ & $15230 \pm 75$ & $\begin{array}{l}16645-16454(1 \sigma) \\
16739-16361(2 \sigma)\end{array}$ & $-19,9$ & 5.1 & 3.3 \\
\hline P-26133 & $\begin{array}{c}2 \text { (fouilles } \\
\text { Bouvier), carré } \\
\text { C9 }\end{array}$ & $\begin{array}{l}\text { os } \\
\text { (fragment } \\
\text { d'humérus) } \\
\text { de bovidé }\end{array}$ & $\begin{array}{l}\text { collecté par J.-M. } \\
\text { Bouvier (fouilles 1966- } \\
\text { 1972) ; sélectionné et } \\
\text { soumis par L. Fontana en } \\
\text { octobre 2009 }\end{array}$ & \multicolumn{2}{|c|}{ échantillon sans collagène } & - & - & - \\
\hline P-26134 & \begin{tabular}{|c|}
3 (fouilles \\
Bouvier), carré \\
BB9
\end{tabular} & $\begin{array}{l}\text { dent } \\
\text { d'équidé }\end{array}$ & $\begin{array}{l}\text { collecté par J.-M. } \\
\text { Bouvier (fouilles 1966- } \\
\text { 1972) ; sélectionné et } \\
\text { soumis par L. Fontana en } \\
\text { octobre 2009 }\end{array}$ & \multicolumn{2}{|c|}{ échantillon sans collagène } & - & - & - \\
\hline $\begin{array}{l}\text { OxA- } \\
22328\end{array}$ & \begin{tabular}{|c|}
4 (fouilles \\
Bouvier), carré \\
C7
\end{tabular} & $\begin{array}{l}\text { dent } \\
\text { d'équidé }\end{array}$ & $\begin{array}{l}\text { collecté par J.-M. } \\
\text { Bouvier (fouilles 1966- } \\
\text { 1972) ; sélectionné et } \\
\text { soumis par L. Fontana en } \\
\text { octobre 2009 }\end{array}$ & $19130 \pm 110$ & $\begin{array}{l}21269-20914(1 \sigma) \\
21476-20770(2 \sigma)\end{array}$ & $-20,7$ & 2.8 & 3.2 \\
\hline $\begin{array}{l}\text { OxA- } \\
22329\end{array}$ & \begin{tabular}{|c|}
5 (fouilles \\
Bouvier), carré \\
BB8
\end{tabular} & $\begin{array}{l}\text { os } \\
\text { (fragment } \\
\text { de tibia) de } \\
\text { Renne }\end{array}$ & $\begin{array}{l}\text { collecté par J.-M. } \\
\text { Bouvier (fouilles 1966- } \\
\text { 1972) ; sélectionné et } \\
\text { soumis par L. Fontana en } \\
\text { octobre 2009 }\end{array}$ & $15805 \pm 80$ & $\begin{array}{l}17208-16995(1 \sigma) \\
17342-16913(2 \sigma)\end{array}$ & $-19,3$ & 4.2 & 3.2 \\
\hline
\end{tabular}

Tableau 2: Caractéristiques des échantillons datés et résultats des mesures C14.

Table 2: Characteristics of the osseous samples submitted for $14 \mathrm{C}$ dating and results of the measurements obtained.

Cet ensemble inférieur à La Chaire-à-Calvin se situerait, au sein de la chronologie qui se dégage actuellement dans le Sud-Ouest de la France, après le Badegoulien (dont la chronologie se resserre, entre 19000/18500 et $17500 / 16500 \mathrm{BP})$ et le Magdalénien inférieur à microlamelles à dos et/ou débitages lamellaires "spéciaux " (Bertonne, Orville, antérieur à 16500/16000 BP) (cf. Langlais, 2007, p. 309-313, 342, annexe 1, p. 457; Pétillon et al., 2016, fig. 3, p. 6). Il correspondrait à un Magdalénien moyen relativement ancien (cf. Demars; Djindjian) ou Magdalénien " pré-classique " (Ladier et Langlais, 2014). Sur un fonds technologique classique du Magdalénien moyen (i.e. grandes lames débitées au percuteur tendre et belles lamelles à dos épais et parfois tronquées), on aurait une phase inférieure (correspondant aux niveaux 6 et 5-2 de Bouvier) dont les traits restent encore à déterminer précisément et une phase supérieure (= niveau 5-1 et partie de 4 de Bouvier), caractérisée notamment par des lamelles scalènes.

\section{Niveau 4}

La nouvelle date de $19130 \pm 110$ B.P. (23220-22 860 cal. B.P. à $1 \sigma$ ) pour le niveau 4 (fouilles Bouvier) est très ancienne par rapport à l'attribution que nous faisons de cet horizon archéologique. Elle est, en outre, plus ancienne que les âges obtenus pour des objets situés stratigraphiquement plus bas. Ce fait est avéré par la position de cette pièce datée, une des quelques côtées, sur le report de projection (fig. 1B). La dent de cheval en question se situe en partie médiane de l'unité lithostratigraphique I, parmi les pièces les plus hautes en altitude de celles rattachées par Bouvier à sa couche 4 . Or, le contenu technologique de la couche 4 est bien magdalénien (Morala, 2007). Cette mesure radiométrique ne date donc ni l'industrie ni cette couche 4 . On peut donc être amené à la rejeter.

Toutefois, nous pouvons proposer une explication pour rendre compte de cette datation a priori aberrante. La dent d'équidé sélectionnée pourrait correspondre à une pièce remaniée, qui serait alors intrusive. Il est en effet possible, comme l'avait déjà envisagé Alimen en son temps (Alimen, 1950, p. 288), que des pièces anciennes aient pu être localement incorporées dans le cryosol et expulsées à son sommet. Ce reste faunique pourrait alors témoigner d'une occupation solutréenne sur le site. Cette hypothèse n'est pas à exclure, d'autant que David dit bien qu'il a trouvé de la faune avec peu ou pas de Saïga dans son niveau profond (faune pléniglaciaire?). La présence, aux côtés des outils solutréens recueillis 
au cours des fouilles anciennes, d'ossements de même âge accréditerait non pas l'interprétation de pièces ramassées par les Magdaléniens, comme l'ont pensé D. de SonnevilleBordes, J.-M. Bouvier et A. Debénath, mais plutôt celle de vestiges d'une occupation du site par les Solutréens, ainsi que l'a compris P. David, lors des premières fouilles du gisement. Une occupation solutréenne en place pourrait avoir existé dans l'abri et, plus discrètement, dans la partie centrale du talus (zones fouillées par P. David, notamment), mais pas dans la zone de fouille des Bordes et de Bouvier, qui pourrait être à la périphérie de l'occupation principale. De ce fait, certains éléments caractéristiques de la chaîne opératoire lithique sont totalement absents : hormis quelques produits finis solutréens (fragments de feuilles de laurier et de pointes à cran), ni éclats de façonnage/retouche solutréenne, ni supports de pointe à cran du Solutréen supérieur, ni préformes de feuille, n’ont été exhumés dans cette zone du gisement, comme F. Bordes et J.-M. Bouvier l'avaient déjà remarqué.

Cette interprétation aurait pour corollaire que la mise en place des dépôts s'est accompagnée d'une " contamination " de la série par redistribution des pièces et nous heurte à la question de l'appréciation de l'impact des agents de sédimentation sur la préservation des niveaux archéologiques, question que nous ne pouvons pas traiter par défaut de données adéquates.

\section{Niveau 1}

La date du niveau 1, de 15230 BP (18590-18400 cal. B.P. à $1 \sigma)$, soulève plus de questions que de réponses, parce qu'elle fait clairement apparaitre les incertitudes qui subsistent encore concernant les caractéristiques et attributions chrono-culturelles des niveaux supérieurs de La Chaire-àCalvin, mais aussi parce qu'elle se cale mal avec les données actuelles de l'évolution régionale.

Si cette datation du niveau 1 s'avèrait correcte, il faudrait reconnaître qu'elle témoignerait d'une chronologie relativement courte sur le talus ("talus-témoin Est»); en effet, elle est à peine plus récente que les dates issues de la base de la séquence. Elle est en tout cas cohérente avec la présence de Saïga dominante, associée au Dryas ancien, dans toute la partie supérieure de la stratigraphie et elle permet d'encadrer assez précisément le cryosol. On serait en présence d'une phase du Magdalénien moyen sans lamelles scalènes. Il faudrait alors envisager que des niveaux plus récents (notamment du Magdalénien supérieur, présents dans l'abri) ne soient pas présents dans cette partie du site fouillée par les Bordes et Bouvier. Par contre, cette datation pourrait également refléter une composante ancienne redistribuée au cours de l'édification du dépôt. Pourtant, dans l'état actuel des travaux en cours, notamment sur la couche 3, il ne semble guère y avoir de traces de contamination des niveaux inférieurs vers le haut, sous la forme de lamelles scalènes par exemple (comm. pers., J. Pelegrin; analyses I. Seo).

Au demeurant, la pièce datée est emprunte d'une certaine incertitude dans son positionnement stratigraphique. Comme le montre la figure $1 \mathrm{~B}$, la couche 1 reconnue par J.-M. Bouvier est assez bien circonscrite au niveau du replat prolongeant l'abri, où elle coiffe les dépôts. Mais elle l'est beaucoup moins au niveau du talus où des pièces attribuées à la couche 1 ont été côtées sur une épaisseur d'un mètre, les plus basses étant au contact du cryosol (rangée 12), voire bien en-deçà (rangée 11); c'est-à-dire que ces pièces attribuées à la couche 1 sur le talus se placent en relais latéral d'éléments des couches 4, 5 ou 6 . On comprend ces incertitudes comme le fait que les couches reconnues par J.-M. Bouvier ont été définies sur des critères lithologiques exprimés au-devant de l'abri, mais relayés par un ensemble massif au niveau du talus; ce qui se traduit par des pièces rattachées à la couche $1 \mathrm{ou}$, plus prudemment, sans rattachement stratigraphique.

On ne saurait donc exclure que la date obtenue pour la couche 1 ne date non pas le sommet du remplissage, mais la base de l'ensemble archéologique supérieur (Unité I), voire le sommet de l'unité archéologique inférieure (Unité II). Si elle ne peut être rejetée, cette hypothèse ne semble toutefois pas la plus probable, eu égard au fait que proportionnellement peu de pièces rattachées à la couche 1 sont issues du talus. Quoi qu'il en soit de cette incertitude, un point reste acquis : le site ne livre, à ce jour, aucune date témoignant d'une occupation plus récente que la première phase du Magdalénien moyen.

De fait, un point délicat à traiter concerne la corrélation de ces niveaux supérieurs sur le talus avec la frise. G. Pinçon et son équipe, à la suite d'une étude comparative de l'art pariétal entre ce site et le Roc-aux-Sorciers, à Angles-sur-l'Anglin (Vienne), sont convaincus de la proche contemporanéité de ces deux productions artistiques et ont maintenant publié plusieurs notes exposant leurs arguments dans ce sens (Bourdier et al. 2008; Pinçon, 2010; Pinçon et Bourdier, 2009; Pinçon et al., 2008). Par ailleurs, les sculptures du Roc-aux-Sorciers semblent assez précisément datées du Magdalénien moyen, associé au « groupe LussacAngles ». Cette phase régionale est actuellement datée entre 14700 et 14100 B.P. (non calibré) (Delage, 2013). Si l'on continue d'accepter une telle attribution chronologique, la frise serait plus récente et ne pourrait être corrélée à aucun des niveaux archéologiques supérieurs (= 4 à 1 des fouilles Bouvier) reconnus dans la zone du talus fouillée par les Bordes et Bouvier. Par contre, si l'on admet que certains des niveaux supérieurs soient contemporains de la frise, il 
faudrait remettre en cause l'attribution chronologique du groupe Lussac-Angles; d'autant plus que l'on peut raisonnablement se poser des questions sur les connaissances que l'on a actuellement de l'âge précis de cette phase du Magdalénien moyen (cf. Langlais et al., 2010). Il faudrait alors envisager de vieillir de 500 à 1000 ans cet épisode.

Même si cette date du niveau 1 participe donc de ces différents questionnements, elle n'est pas suffisante et confirme la nécessité d'obtenir de nouvelles dates, sur ce gisement précis et à l'échelle régionale, pour cette phase spécifique du Magdalénien.

\section{Conclusion}

Deux datations radiométriques étaient connues jusqu’à ces dernières années, mais elles s'accordaient mal avec les interprétations fondées sur une chronologie relative assez approximative proposées par J.-M. Bouvier et A. Debénath. De nouvelles mesures radiocarbone étaient donc nécessaires. Dans le cadre d'un nouveau programme répondant à ces objectifs, cinq échantillons osseux ont été envoyés pour datation au Laboratoire de datation d'Oxford et trois mesures ont pu être obtenues.

La nouvelle datation du niveau 5 (fouilles Bouvier) s'accorde bien avec une autre produite récemment pour le niveau 6 (fouilles Bouvier) : $16020 \pm 80$ B.P. (OxA-12053/ Ly-2098), montrant que l'écart chronologique entre les niveaux 6 et 5 est relativement faible et qu'ils peuvent appartenir à un seul et même ensemble culturel, attribué à la première partie du Magdalénien moyen. La date du niveau 4 est trop ancienne par rapport à cet horizon archéologique. Elle serait soit aberrante et à rejeter, soit intrusive et reflèterait la présence d'une occupation solutréenne dans une autre partie du site. La datation du niveau 1 pourrait être correcte, mais elle témoigne alors d'une chronologie relativement courte sur le talus et implique que des niveaux encore plus récents du Magdalénien ne sont pas présents dans cette partie du site, ou reflète un problème de lecture stratigraphique lors des fouilles anciennes. Elle pose, dans tous les cas, des problèmes de corrélation des niveaux supérieurs du talus avec la frise.

Ce travail de datation est donc une contribution importante à la connaissance de la séquence chrono-culturelle de La Chaire-à-Calvin. Toutefois, il a produit toute une série de nouvelles questions qui ne pourront être résolues que par une nouvelle campagne de datation des différents niveaux accompagnée d'une ré-évaluation de l'archéostratigraphie du site.

\section{Remerciements}

Nous remercions vivement Thomas F.G. Higham et le Laboratoire de Datation d'Oxford sans qui le présent projet n'aurait pu aboutir. Nous tenons ensuite à exprimer notre gratitude à Alain Queffelec, Thibault Morala, Jacques Pelegrin et Laure Fontana pour leur aide à la préparation de ce travail, ainsi qu'à Jean-François Tournepiche et au musée des BeauxArts d'Angoulême, au Service Patrimoine du Conseil départemental de la Charente, et au Service régional de l'Archéologie de Poitou-Charentes pour leur soutien.

\section{Bibliographie}

Alimen H., 1950 - "Indications climatiques dans les couches archéologiques d'un abri (sol polygonal de Mouthiers, Charente) ", Bulletin de la Société Préhistorique Française, 47, p. 286-288.

Alimen H., David P., 1949 - «Cryoturbations dans des couches archéologiques de la Charente et du Périgord ", Comptes rendus hebdomadaires des séances de l'Académie des sciences, 229, p. 1246-1247.

Bertran P., Texier J.-P., 1997 - «Géoarchéologie des versants : les dépôts de pente ", in Bravard J.-P. et Presteau M. (éd.), Dynamique du paysage : entretiens de géoarchéologie, Châtillonsur-Chalaronne, Documents d'Archéologie en Rhône-Alpes, p. 59-86.

Bertran P., Coussot P., 2004 - Dépôts de pente continentaux : dynamique et faciès, P. Bertran (éd.), Orléans, Éditions du BRGM, p. 132-151.

Bourdier C., Fuentes O., Hamon G., Pinçon G., 2008 "Technologies 3D appliquées à la sculpture pariétale magdalénienne ", in Buchsenschutz O. (éd.), Images et relevés archéologiques, de la preuve à la démonstration, Paris, Éditions du CTHS, p. 123-142. (132 e congrès national des sociétés historiques et scientifiques, Arles 2007).

Bourgueil B., Moreau P., Vouve J., 1970 - Notice explicative de la carte géologique d'Angoulême au 1/50 000. Orléans, Bureau de Recherches géologiques et minières, 20 p. (Carte $n^{\circ} 709$ ).

Bouvier J.-M., 1968 - "Godet en stéatite et collier magdaléniens de la Chaire à Calvin, Mouthiers (Charente) ", Mémoires de la Société archéologique et historique de la Charente, 1968, p. 65-72.

Bouvier J.-M., 1969 - « Existence de Magdalénien supérieur sans harpon : preuves stratigraphiques ", Comptes Rendus de l'Académie des Sciences de Paris, 268, p. 2865-2866.

Bouvier J.-M., 1971 - «L'outillage osseux magdalénien de la Chaire-à-Calvin, Mouthiers (Charente) ", Mémoires de la Société Archéologique et Historique de la Charente, 1971, p. 163-177. 
Bouvier J.-M. 1976 - «La Chaire à Calvin, Mouthiers (Charente) : données et problèmes ", in Rigaud J.-Ph., Vandermeersch B. (éd.), Livret-guide de l'excursion A4-Sud-Ouest (Aquitaine et Charente), IX ${ }^{\mathrm{e}}$ congrès UISPP, Nice, p. 133-136.

Bouvier J.-M., 1977 - Un gisement préhistorique : La Madeleine, Périgueux, Pierre Fanlac, 88 p.

Bouvier J.-M., Cremadès M., 1989 - Les sculptures magdaléniennes de la Chaire à Calvin (Mouthiers, Charente). Lectures complémentaires, Bulletin de la Société Historique et Archéologique du Périgord, CXVI, p. 73-85.

Bouvier J.-M., Debénath A., 1969 - « La Chaire à Calvin (Mouthiers, Charente). Etude sédimentologique : note préliminaire", Quaternaria, XI, p. 215-226.

Brock F., Higham T., Ditchfield P., Bronk Ramsey C., 2010 - "Current Pretreatment Methods for AMS Radiocarbon Dating at the Oxford Radiocarbon Accelerator Unit (ORAU) » Radiocarbon, 52/1, p. 103-112.

Bronk Ramsey C., Higham T., Leach P., 2004 - « Towards HighPrecision AMS, Progress and Limitations ", Radiocarbon, 46/1, p. 17-24.

Coplen T.B., 1994 - Reporting of Stable Hydrogen, Carbon, and Oxygen Isotopic Abundances (Technical Report), Pure and Applied Chemistry, 66, p. 273-276.

DAvid P., 1928 - «Abri sous roche dit de la "Chaire à Calvin” ou de la "Papeterie". Commune de Mouthiers (Charente) ", in Congrès de l'Association Française pour l'Avancement des Sciences, $52^{\mathrm{e}}$ session, La Rochelle, Laval, Imprimerie Barnéoud, p. 429431.

DAvid P., 1929 - «Frise de l'abri sous roche dit "de la Chaire à Calvin" ou de la "Papeterie", Commune de Mouthiers (Charente) ", in Congrès de l'Association Française pour l'Avancement des Sciences, 53 ${ }^{\mathrm{e}}$ session, Le Havre, Laval, Imprimerie Barnéoud, p. 1-4.

DAvid P., 1957 - « La Chaire-à-Calvin, commune de Mouthiers (Charente) ", in Congrès Préhistorique de France, ComptesRendus de la $X V^{e}$ session, Poitiers-Angoulême, 15-22 juillet 1956, Paris, Bureaux de la Société Préhistorique Française, p. 127-129.

DAvid P., 1963 - «Sculptures paléolithiques de l'abri de la Chaireà-Calvin, Commune de Mouthiers (Charente) ", Annales de Paléontologie, XLIX, p. 173-177.

DeBÉNATH A., 1974 - Recherches sur les terrains quaternaires charentais et les industries qui leur sont associées, Bordeaux, Université de Bordeaux I, 2 vols. Thèse de Doctorat d'État.

DebénAth A., 2006 - Néandertaliens et Cro-Magnons, les temps glaciaires dans le bassin de la Charente, Paris, Le Croît Vif, 356 p.

Debénath A. 2014 - Histoire de la préhistoire en Charentes, Saintes, Le Croît Vif, coll. "Documentaires ", 392 p.

Delage C., 2010 - « La Chaire-à-Calvin, Mouthiers-sur-Boëme, Charente ", in Livret-guide des Excursions A1 et A2 (PoitouCharentes). XXVII Congrès Préhistorique de France, Bordeaux-
Les Eyzies, 31 mai-5 juin 2010. Poitiers, Service Régional de l'Archéologie/Direction Régionale des Affaires Culturelles, p. 33-42.

Delage C., 2013 - "De la "pointe de sagaie" à la "Culture de Lussac-Angles", il y a plus qu'un pas. Argumentaire ", Bulletin de la Société d'Etudes et de Recherches Préhistoriques des Eyzies, 62 (Travaux de 2012), p. 23-48.

Delage C., Lenoble A., Queffelec A., Argant J., Dayet L., Daniel F., Platel N., Santallier D., Fontana L., 2010 - "Mouthiers-sur-Boëme, La Chaire-à-Calvin ", in Bilan scientifique régional de Poitou-Charentes 2009, Poitiers, Service Régional de l'Archéologie, Direction Régionale des Affaires Culturelle, p. 42-43.

Delage C., Porte A., 2015 - «Jean Calvin à Mouthiers-surBoëme? Réflexions sur l'origine du nom Chaire-à-Calvin ", Bulletins et Mémoires de la Société Archéologique et Historique de la Charente, $168^{\mathrm{e}}$ année (2012), p. 7-24.

Dujardin V., Tymula S., 2005 - "Relecture chronologique de sites paléolithiques et épipaléolithiques anciennement fouillés en Poitou-Charentes ", Bulletin de la Société Préhistorique Française, 102/4, p. 771-788.

Evin J., Marechal J., Marien G., 1983 - "Lyon Natural Radiocarbon Measurements IX ", Radiocarbon, 25/1, p. 59-128.

Gasche H., Tunca Ö., 1981 - Guide de classification et de terminologie archéostratigraphiques : définition et principes, PICG 146, Groupe de Travail 2. 54 p.

LAdier E., LANGLAis M., 2014 - « Bilan sur les occupations magdaléniennes ", in LAdier E. (éd.), L'abri Gandil à Bruniquel (Tarn-et-Garonne) : un campement magdalénien du temps de Lascaux, Cressenac, Association Préhistoire du Sud-Ouest, p. 259-263. (Préhistoire du Sud-Ouest Supplément 13).

LANGLAIs M., 2007 - Dynamiques culturelles des sociétés magdaléniennes dans leurs cadres environnementaux. Enquête sur 7000 ans d'évolution de leurs industries lithiques entre Rhône et Ėbre, Toulouse, Université de Toulouse II, Thèse de Nouveau Doctorat.

Langlais M., Pétillon J.-M., Beaune S.A. de, Cattelain P., Chauvière F.-X., Letourneux C., Szmidt C., Bellier C., Beukens R., David F., 2010 - «Une occupation de la fin du Dernier Maximum glaciaire dans les Pyrénées : le Magdalénien inférieur de la grotte des Scilles (Lespugue, Haute-Garonne) ", Bulletin de la Société Préhistorique Française, 107/1, p. 5-51.

LENOBLE A., 2005 - Ruissellement et formation des sites préhistoriques : référentiel actualiste et exemples d'application au fossile, Oxford, Archaeopress, 217 p. (BAR International Series 1363).

Morala T., 2007 - Etude technologique et économique de l'industrie lithique de la couche 4 de l'abri de la Chaire-à-Calvin (Mouthiers-sur-Boëme, Charente) : contribution à la caractérisation du Magdalénien charentais, Bordeaux, Université de Bordeaux I, 212 p. Mémoire de Master 2. 
Pétillon J.-M., Laroulandie V., Costamagno S., Langlais M., 2016 - "Testing Environmental Determinants in the Cultural Evolution of Hunter-Gatherers: A Three-Year Multidisciplinary Project on the Occupation of the Western Aquitaine Basin during the Middle and Upper Magdalenian (19-14 kyr cal BP) ", Quaternary International, 414, p. 1-8.

Pinçon G., 2010 - "L'art pariétal de La Chaire-à-Calvin (Mouthiers-sur-Boëme, Charente) ", in Buisson-Catil J. et Primault J. (éd.), Préhistoire entre Vienne et Charente. Hommes et sociétés du Paléolithique, Chauvigny, Association des Publications Chauvinoises, p. 461-475. (Mémoire XXXVIII). Pinçon G., Bourdier C., 2009 - « Nouvelle interprétation d'une sculpture pariétale de la Chaire-à-Calvin (Charente, France) : apport de la technologie 3D ", International Newsletter on Rock Art, 54, p. 11-16.

Pinçon G., Bourdier C., Fuentes O., 2008 - « Les sculptures pariétales magdaléniennes du Roc-aux-Sorciers (Vienne) et de la Chaire-à-Calvin (Charente) : oeuvres d'un groupe culturel ou d'un seul et même artiste? ", in Vergnieux R. et Delevoie C. (éd.), Actes du Colloque Virtual Retrospect 2007, Bordeaux, Ausonius, p. 13-19. (Archéovision, 3).

Reimer P.J., Bard E., Bayliss A., Beck J.W., Blackwell P.G., Ramsey C.B., et al., 2013 - "IntCal13 and Marine13
Radiocarbon Age Calibration Curves 0-50,000 Years cal BP ", Radiocarbon, 55/4, p. 1869-1887.

Sonneville-Bordes D. de, 1965 - « L'abri de la Chaire-à-Calvin, Mouthiers (Charente) ", Bulletin de l'Association française pour l'étude du Quaternaire, 1965/3-4, p. 193-197.

Sonneville-Bordes D. DE, 1987 - «Observations sur le Magdalénien de la Chaire-à-Calvin, à Mouthiers (Charente) ", in Vandermeersch B. (éd.), Préhistoire de Poitou-Charentes: problèmes actuels. Actes du $111^{\mathrm{e}}$ Congrès national des Sociétés savantes, Poitiers 1986, Paris, Éditions du CTHS, p. 157-185.

Stuiver M., Polach H.A., 1977 - « Discussion: Reporting of C-14 Data ", Radiocarbon, 19/3, p. 355-363.

Texier J.-P., 2000 - « À propos des processus de formation des sites préhistoriques ", Paléo, 12, p. 379-386.

Tremeau de Rochebrune A., 1865 - « Mémoires sur les restes d'industrie appartenant aux temps primordiaux de la race humaine, recueillis dans le département de la Charente ", Mémoires de la Société des Antiquaires de l'Ouest, XXX, p. 57-182.

Van Vliet-Lanoë B., 2005 - La planète des glaces, Paris, Vuibert, $470 \mathrm{p}$.

Zusammenfassung: Neue Radiokarbondatierungen des Magdaleniens von La Chaire-à-Calvin (Mouthiers-sur-Boëme, Charente) - Der magdalénienzeitliche Fundplatz von La Chaire-à-Calvin (Mouthiers-sur-Boëme, Charente) ist seit langer Zeit bekannt. Jetzt, nach rund 150 Jahren archäologischer Forschungen, ist festzustellen, dass die chronostratigraphischen Interpretationen verschiedener Forschergenerationen weder untereinander korrespondieren noch mit den beiden vorliegenden Radiokarbondatierungen in Übereinstimmung zu bringen sind. Vor diesem Hintergrund hat ein neu gestartetes Programm von Radiokarbondatierungen an der Schichtenfolge drei neue Daten erbracht. Dieser Beitrag stellt die neu erzielten Datierungsergebnisse vor und diskutiert die daraus abzuleitenden Schlussfolgerungen in Bezug auf die archäologische Stratigraphie des Fundplatzes.

Resumen: Nuevas fechas radiocarbónicas del magdaleniense de la Chaire-à-Calvin (Mouthiers-sur-Boëme, Charente) - El yacimiento magdaleniense de La Chaire-à-Calvin (Charente) es conocido desde antiguo. Sin embargo, tras 150 años de investigación, las interpretaciones cronoestratigráficas propuestas por varias generaciones de investigadores no concuerdan entre si, ni con las dos fechas radiocarbónicas disponibles. En este contexto, un nuevo programa de medidas radiométricas del relleno ha permitido obtener tres nuevas fechas. Esta nota expone los resultados y analiza su validez dada la secuencia del yacimiento.

Schlüsselwörter: La Chaire-à-Calvin, Magdalénien, Radiokarbondatierungen.

Palabras clave: La Chaire-à-Calvin, Magdaleniense, fechas radiocarbónicas. 